\title{
Surface Heat Island in Shanghai and Its Relationship with Urban Development from 1989 to 2013
}

\author{
Liang Chen, ${ }^{1,2}$ Rong Jiang, ${ }^{2}$ and Wei-Ning Xiang ${ }^{2}$ \\ ${ }^{1}$ School of Geographic Sciences, East China Normal University, Shanghai 200241, China \\ ${ }^{2}$ Shanghai Key Lab for Urban Ecological Processes and Eco-Restoration, School of Ecological and Environmental Sciences, \\ East China Normal University, Shanghai 200241, China
}

Correspondence should be addressed to Liang Chen; lchen@des.ecnu.edu.cn

Received 11 August 2015; Accepted 29 September 2015

Academic Editor: Sergio M. Vicente-Serrano

Copyright (C) 2016 Liang Chen et al. This is an open access article distributed under the Creative Commons Attribution License, which permits unrestricted use, distribution, and reproduction in any medium, provided the original work is properly cited.

\begin{abstract}
The continuous expansion of impervious artificial surfaces in cities has significantly influenced the urban thermal environment. This paper examines the spatiotemporal variation of the diurnal surface urban heat island (SUHI) in Shanghai from 1989 to 2013 , a period during which the city has experienced drastic development changes. A remote sensing approach was taken to derive the spatial patterns of Shanghai's land surface temperature (LST) from Landsat Thematic Mapper (TM) images and Operational Land Imager (OLI) data. The LST pattern was further classified into five LST classes to look at the relative SUHI intensity level across the whole city. Spatial analyses, namely, spatial association and centroid movement analysis, were conducted to reveal the trends of LST changes at both local and holistic scales. To understand the potential drivers for the present spatiotemporal variation of SUHI, different indicators including land use change, population density, night light data, and vegetation were analyzed and compared with LST changes. Based on the quantitative analysis and the socioeconomic context of Shanghai, "heating up" regions were identified, possible reasons for such SUHI variation were summarized, and districts that are most vulnerable to extreme heat conditions were projected. In terms of implication for urban development, planning and design recommendations were suggested to improve the urban thermal environment in Shanghai.
\end{abstract}

\section{Introduction}

More than half of the world's populations now live in cities (Population Reference Bureau, 2009). And it is projected that by 2025 the world's population will reach 8 billion, of which 60 percent or nearly 5 billion are urban population [1]. With more people coming to live and work in cities, the expansion of "built-up" area, or impervious surfaces to be more specific, seems to be inevitable. In contrast with natural landscape, cities have heterogeneous surface characteristics and complex energy fluxes, which affect the equilibrium for exchanges of energy and water vapor and result in excessive heat accumulation in urban areas [2]. One of the most widely observed results of this complex impact is the urban heat island effect (UHI) (e.g., $[3,4]$ ), which refers to the phenomenon that urban area has higher air or surface temperature than the surrounding suburban or rural area. UHI is closely related with urban living quality and people's health, especially in hot seasons and adverse climatic conditions [5, 6]. Therefore the study of UHI and its pattern, dynamics, possible drivers, and impacts has been a continuous pursuit for climatologists and urban planners $[3,7]$.

UHI can be reflected by atmospheric or surface temperature. Traditional observation methods such as meteorological stations look at the air temperature, and point data collected from station networks can be interpolated to describe the air temperature difference at relatively small scales. Recent advances in satellite remote sensing techniques make it possible to measure UHI by surface temperature, that is, the surface urban heat island (SUHI) [8]. Compared with traditional in situ observation methods with limited spatial coverage and poor spatial resolution, remote sensing method has its biggest advantage in that it provides continuous coverage, high integrity, and real-time data acquisition over large areas and can therefore reveal the spatial and temporal variability of the urban thermal environment at both macro 
and micro scales [7-9]. As the air temperature in UHI, the land surface temperature (LST) is the most important indicator that quantifies SUHI. Many types of satellite-derived thermal data have been used, including Very High Resolution Radiometer (VHRR) data, Heat Capacity Mapping Mission (HCMM) data, Landsat Thematic Mapper (TM) data, Landsat Enhanced Thematic Mapper plus (ETM+) data, Moderate Resolution Imaging Spectroradiometer (MODIS) data, and Operational Land Imager (OLI) data [10-16]. The spatial accuracy ranges from the scale of $10 \mathrm{~km}$ to $10 \mathrm{~m}$. The LSTs for different seasons have been retrieved [17, 18], and both diurnal and nocturnal SUHI have been examined $[19,20]$.

A number of algorithms have been developed to retrieve LSTs from remote sensing data. Notable examples include the single-window algorithm [21] and the split-window algorithm [22]. The universal single-channel (USC) algorithm [21] is a commonly used approach to estimate LST based on Landsat TM thermal-infrared remote sensing image data because of its relatively simple data requirements and high accuracy. The USC algorithm only requires one thermalinfrared channel and uses the same equation and coefficients for different sensors, whereas split-window algorithms require simultaneous data from at least two sensor channels. Also, compared with other single-channel algorithms, the USC algorithm only requires the atmospheric moisture content and land surface emissivity as parameters. These advantages make USC widely applied in SUHI studies in cities across the world [23-25].

Shanghai is one of the biggest and most rapidly developing cities in China. It has experienced drastic urban development since China's economic reform in the early 1980s. Meanwhile, developing policies and strategies put into effect at different phases lead to different development stages. For example, the development of Pudong district, which started in the early 1990s, established the Lujiazui area which is now the downtown CBD, the industrial parks in the northern and southern urban fringe have accelerated the growth of the local residential area before the 2000s, and the 2010 Expo also brought a lot of open spaces and urban greening to the city. Therefore, how the city's urban thermal environment, that is, the SUHI, has evolved at different development stages became an interesting question. On the other hand, although the study of SUHI in Shanghai has drawn increasing attention, previous studies mostly cover a limited time such as single year, or 5 to 10 years, with a focus on a particular development stage [26-28]. The evolution of the SUHI of Shanghai over a long time period that covers different socioeconomic development stages remains a poorly studied area.

The objective of the study is twofold. Firstly, it examines the spatiotemporal characteristics of the SUHI change in Shanghai, as quantified by the LST change from 1989 to 2013. Remote sensing data of Landsat TM and OLI images were used to retrieve the summer time diurnal LST, which was further classified to show the SUHI intensity. Spatial analyses including spatial association and centroid movement analysis were conducted to derive the spatial pattern and dynamics of SUHI change both on the local and on the holistic spatial scales. Secondly, it relates the SUHI change with the socioeconomic context of Shanghai, including land use change,

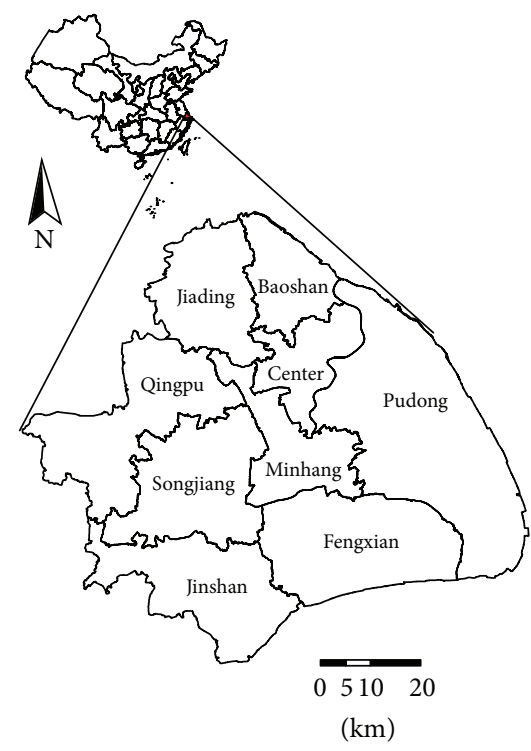

FIGURE 1: Location of the study area.

population density, urban greening, and human activities, and investigates potential drivers of such SUHI change and discusses possible impacts of the thermal environment on urban living quality. "Heating up" regions were identified, and districts that are most vulnerable to extreme heat conditions were projected. Based on the results and discussion, this paper further proposes planning recommendations for urban development that will be more responsive to future climatic variations in Shanghai.

\section{Methods}

2.1. Study Area. Shanghai $\left(30^{\circ} 40^{\prime} \mathrm{N} \sim 31^{\circ} 53^{\prime} \mathrm{N}, 120^{\circ} 51^{\prime} \mathrm{E} \sim\right.$ $122^{\circ} 12^{\prime} \mathrm{E}$ ) is located on the alluvial terrace of the Yangtze River delta. Shanghai's average elevation is about $4 \mathrm{~m}$ above sea level. It has a northern subtropical monsoon climate, with a mean annual temperature of $17.2^{\circ} \mathrm{C}$, and monthly maximum and minimum mean temperatures of $30.2^{\circ} \mathrm{C}$ in July and $1.9^{\circ} \mathrm{C}$ in January, respectively. The mean annual precipitation averages $902.9 \mathrm{~mm}$, with more than $60 \%$ of the rainfall occurring during May and September. The city's administrative boundaries cover a total terrestrial area of $6340.5 \mathrm{~km}^{2}$ excluding estuary waters. In terms of socioeconomic levels, the Shanghai metropolitan area consists of 16 districts, namely, Huangpu, Xuhui, Changning, Jing'an, Putuo, Hongkou, Zhabei, Yangpu, Pudong, Minhang, Baoshan, Jiading, Jinshan, Songjiang, Qingpu, and Fengxian, and 1 island, the Chongming Island. According to Shanghai Statistical Yearbook 2014, the city has a total population of 24.15 million, including both permanent and nonpermanent residents.

Because of the scope of the present study, which is the SUHI characteristics of Shanghai, it focuses on the 16 administrative districts of Shanghai, that is, excluding the Chongming Island and the Yangtze River estuary waters between the island and the city. Figure 1 shows the location 
of the study area and the districts of interest. The total study area is approximately $5140.8 \mathrm{~km}^{2}$. The 8 districts of Huangpu, Xuhui, Changning, Jingan, Putuo, Hongkou, Zhabei, and Yangpu are located in inner city and are districts with high socioeconomic levels, that is, high residential density and commercial intensity, and therefore were grouped together as the city center. The Pudong district is located on the east bank of the Huangpu River. As mentioned in the previous section, the region along the Huangpu River has experienced booming development since the 1990s, and the area centered by Lujiazui becomes Shanghai's new CBD. The districts of Minhang, Baoshan are urban fringes with increasing developing intensity. Industry infrastructures can be found in these districts, such as the Wujing Chemical Industrial Factory in Minhang, and the Baosteel Steel Manufacturing Corporation in Baoshan. The districts of Jiading, Jinshan, Songjiang, Qingpu, and Fengxian are essentially suburban areas with new towns, as characterized by the relatively low residential and commercial density, and broad farmland and natural landscape. These districts were previously counties and only became Shanghai's administrative districts in recent years.

2.2. Data Source and Retrieval of LST. Cloud-free Landsat TM and OLI images covering Shanghai (rows 038 and 039, path 118) were acquired for 11 August 1989, 2 September 2000, and 13 August 2013. The USC algorithm was used to retrieve LST values from the thermal-infrared band. The procedures given by [21] was adopted:

$$
T_{s}=\gamma\left\{\left[\frac{\left(\varphi_{1} L_{\text {sensor }}+\varphi_{2}\right)}{\varepsilon}\right]+\varphi_{3}\right\}+\delta,
$$

where $T_{s}$ represents the LST, $L_{\text {sensor }}$ represents the thermalinfrared value from the sensor, $\varepsilon$ represents the surface emissivity, and $\gamma$ and $\delta$ are obtained from the linear approximation of Planck's law. Their values were given by

$$
\begin{aligned}
& \gamma=\frac{1}{\left[C_{2} L_{\text {sensor }}\left(\lambda^{4} L_{\text {sensor }} / C_{1}+1 / \lambda\right) / T_{\text {sensor }}^{2}\right]}, \\
& \delta=T_{\text {sensor }}-\gamma L_{\text {sensor }},
\end{aligned}
$$

where $C_{1}$ and $C_{2}$ are the Plank coefficients, $C_{1}=1.19104 \times$ $10^{8} \mathrm{~W} \cdot \mu \mathrm{m}^{4} \cdot \mathrm{m}^{-2} \cdot \mathrm{sr}^{-1}, C_{2}=14387.7 \mu \mathrm{m} \cdot \mathrm{K} ; T_{\text {sensor }}$ is the sensor's pixel temperature in Kelvin; $\lambda$ is the effective wavelength for emitted radiance and is $11.457 \mu \mathrm{m}$ for Landsat TM6 [29]. And $\varphi_{1}, \varphi_{2}$, and $\varphi_{3}$ represent regression parameters related to atmospheric function and were assumed to be dependent only on atmospheric water vapor. Their values were given by (3) as proposed in [30]:

$$
\begin{aligned}
& \varphi_{1}=0.14714 w^{2}-0.15583 w+1.1234 \\
& \varphi_{2}=-1.1836 w^{2}-0.37607 w-0.52894 \\
& \varphi_{3}=-0.04554 w^{2}+1.8719 w-0.39071
\end{aligned}
$$

where $w$ is the water vapor and was calculated based on the ground-level meteorological data. The detailed calculation procedures of these parameters are not the foci of this study
TABLE 1: Threshold values for LST classification. $\mu$ is the average value of LST; std is the standard deviation of LST.

\begin{tabular}{lc}
\hline LST category & Range \\
\hline Very low & $T_{s}<\mu-1$ std \\
Low & $\mu-1$ std $\leq T_{s}<\mu-\operatorname{std} / 3$ \\
Medium & $\mu-\operatorname{std} / 3 \leq T_{s} \leq \mu+\operatorname{std} / 3$ \\
High & $\mu+\operatorname{std} / 3<T_{s}<\mu+1$ std \\
Very high & $T_{s} \geq \mu+1$ std \\
\hline
\end{tabular}

and therefore are not introduced here. Version 4.8 of the ENVI software was used for atmospheric correction before LST calculation.

2.3. Classification of SUHI Intensity and Change Trend. In order to better understand the SUHI intensity, that is, the LST difference between the city center and the surrounding suburban areas, the retrieved LST was classified into five levels according to the deviation from the mean value. The five levels are very low, low, medium, high, and very high. Similar denotations were also used in [26]. Table 1 gives the threshold values for the classification: for example, if a pixel's LST is lower than the mean LST, $\mu$, minus the standard deviation, std, then the SUHI intensity of this pixel will be classified as "very low," and the other threshold values are $\mu-\operatorname{std} / 3, \mu+\operatorname{std} / 3$, and $\mu+1$ std, respectively. Admittedly the current selection of the threshold values is just one possibility out of many alternatives; nevertheless the advantage of using mean value and standard deviation is that the spatial differentials of LST can be revealed regardless of the actual LST values which vary between different years.

LST classification was generated for each year. Numerical values were assigned to the LST classes: very low $=1$, low $=2$, medium $=3$, high $=4$, and very high $=5$. This definition ignores the temperature differences between years and only measures the relative difference between LST classes in each year. The change in LST between two years was calculated based on these assigned values and classified into three trends, namely, large decrease, little change, and large increase. For example, if a pixel changes from very low LST (with the numerical value of 1 ) to very high LST (with the numerical value of 5 ), then the trend value equals $4(5-1)$. Using this procedure a total of nine possible trend values were calculated $(-4,-3,-2,-1,0,1,2,3$, and 4$)$, which were further classified into 3 classes. The classification of trend values and their relationships with LST changes were summarized in Table 2 .

2.4. Spatial Analysis Methods. Two types of spatial analyses were carried out, namely, spatial association and centroid movement analysis. They look at the spatial pattern and dynamics of LST change from the local and holistic scales, respectively.

2.4.1. Spatial Association. Spatial association analysis can provide interpretations of a potential spatial pattern through the assessment of significant local spatial clustering around an individual location. The main significance of spatial 
TABLE 2: The LST changes and their relationships with the three LST trends of large increase, little change, and large decrease. LST values were defined as the following scores: very low $=1$, low $=2$, medium $=3$, high $=4$, and very high $=5$.

\begin{tabular}{lcl}
\hline Trend & Trend value & Change \\
\hline \multirow{2}{*}{ Large increase } & 4 & Very low $\rightarrow$ very high \\
& 3 & Very low $\rightarrow$ high; low $\rightarrow$ very high \\
& 2 & Very low $\rightarrow$ medium; low $\rightarrow$ high; medium $\rightarrow$ very high \\
\hline \multirow{2}{*}{ Little change } & 1 & Very low $\rightarrow$ low; low $\rightarrow$ medium; medium $\rightarrow$ high; high $\rightarrow$ very high \\
& 0 & The same level (i.e., no change) \\
& -1 & Low $\rightarrow$ very low; medium $\rightarrow$ low; high $\rightarrow$ medium; very high $\rightarrow$ high \\
Large decrease & -2 & Medium $\rightarrow$ very low; high $\rightarrow$ low; very high $\rightarrow$ medium \\
& -3 & High $\rightarrow$ very low; very high $\rightarrow$ low \\
\hline
\end{tabular}

association analysis is to explore the spatial dependence and space correlation by establishing the statistics relationship according to spatial location [31]. In exploratory spatial data analysis, the predominant approach to assess the degree of spatial association is based on global statistics such as Moran's $I$ or Geary's $C$ indicators [32], which tends to ignore the potential instability. Recently the focus on local patterns of association which allows local instabilities in overall spatial association has been suggested. In this study, the local indicator of spatial association (LISA) proposed by [31] was adapted to examine the spatial characteristic of LST classes. It was calculated based on the following equations [31]:

$$
\begin{aligned}
& I=\frac{n \sum_{i=1}^{n} \sum_{j=1}^{n} w_{i j}\left(x_{i}-\bar{x}\right)\left(x_{j}-\bar{x}\right)}{\sum_{i=1}^{n} \sum_{j=1}^{n} w_{i j} \sum_{i=1}^{n}\left(x_{i}-\bar{x}\right)^{2}}, \\
& I_{i}=\frac{\left(x_{i}-\bar{x}\right)}{S^{2}} \quad S^{2}=\sum_{j}^{n} \frac{\left(x_{j}-\bar{x}\right)^{2}}{n} .
\end{aligned}
$$

Equations (4) calculate the global indicators (Moran's $I$ ) and LISA, respectively. In the equations, $x_{i}$ and $x_{j}$ are LST class values of the adjacent pixel, $\bar{x}$ is the average of LST class values, $n$ is the number of patterns, $w_{i j}$ is the weight of adjacent pixel, and $S^{2}$ is the variance. By definition, positive $I_{i}$ indicates that a high value is surrounded by a high value, or a low value is surrounded by a low value, while negative $I_{i}$ indicates that a high value is surrounded by a low value or a low value is surrounded by a high value. By combining LISA and local LST class value, 5 types of aggregation pattern were defined, namely, high-high (indicating high LST surrounded by high LST), low-low (indicating low LST surrounded by low LST), low-high (indicating low LST surrounded by high LST), high-low (indicating high LST surrounded by high LST), and not significant (indicating the same grade of LST).

2.4.2. Centroid Movement Analysis. Based on the change trends analysis of LST levels, centroid movement analysis of different LST classes was conducted to examine the characteristics of its spatiotemporal evolution which reflects the changing dynamics of the thermal pattern in Shanghai from 1989 to 2013. The change of a landscape's centroid indicates the areal differentials of its characteristics' change.
That is to say, if a landscape changes evenly for the whole area, then presumably there is only slight change for its centroid's location, whereas the centroid would change largely if the landscape experienced various changes for different locations. In this sense, the centroids of different LST classes were calculated for each year, and the movements of the centroids for all 5 LST classes between different years were drawn on the map to show the spatial migration of the thermal pattern. The centroid coordinates were calculated according to the following formula as suggested by [33]:

$$
\begin{gathered}
X_{c}=\frac{\sum_{i=1}^{n} C_{i} X_{i}}{\sum_{i=1}^{n} C_{i}} ; \\
Y_{c}=\frac{\sum_{i=1}^{n} C_{i} Y_{i}}{\sum_{i=1}^{n} C_{i}},
\end{gathered}
$$

where $X_{c}$ and $Y_{c}$ are the centroid coordinates of a LST class calculated by area-weighted average, $X_{i}$ and $Y_{i}$ are the centroid coordinates of the $i$ pixel with the LST class, $C_{i}$ is the area of pixel $i$, and $n$ is the number of all pixels with the same LST class value.

\section{Results and Analysis}

3.1. Classification and Change Trends of SUHI Intensity. Figure 2 shows the spatial distribution of Shanghai's LST for 1989 (a), 2000 (b), and 2013 (c). The figure shows a clear pattern: areas with very high LST mainly occurred in the central urban area, whereas areas with very low and low LST mostly occurred along the Huangpu River and the urban fringe. Notably the very high LST in areas outside the city center showed a more scattered picture which can be attributed to point sources such as factories in the north of Baoshan and south of Minhang. Another notable feature is the significant expansion of areas with very high LSTs from the urban center from 1989 to 2013. Figure 3 shows the proportion of the land area in different LST classes for each year. It is found that areas with very high LSTs have a stable proportion over the years: $12.57 \%, 16.56 \%$, and $16.54 \%$, which indicates that the city has a substantial proportion that has been constantly dominated by very high LST. In contrast, areas with very low LSTs have significantly increased from $1.34 \%$ to $8.43 \%$ to $17.74 \%$. This suggests that the intraurban 


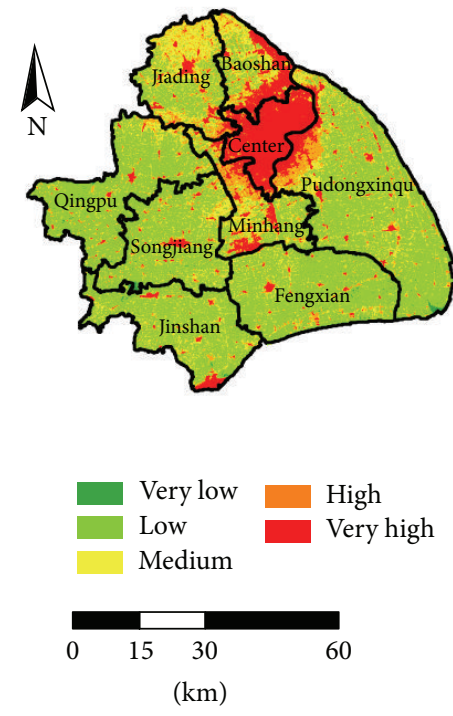

(a)
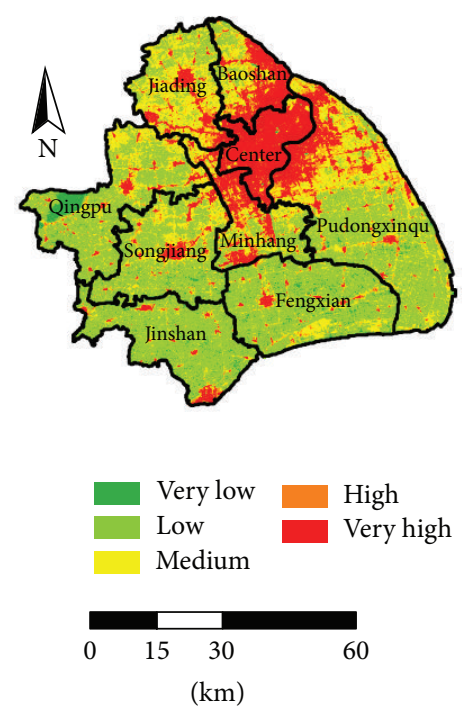

(b)
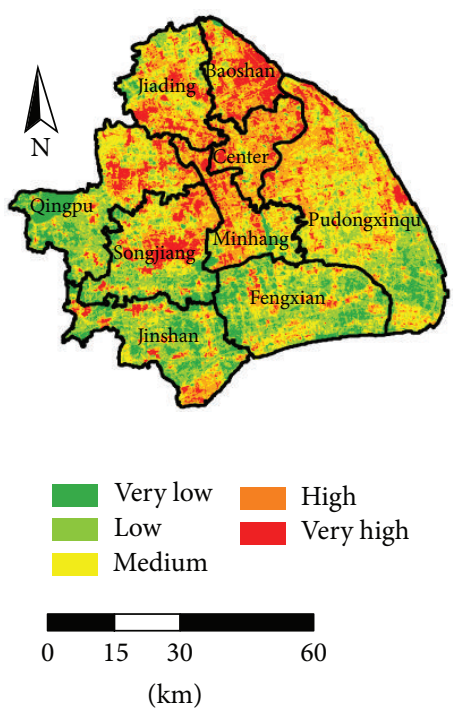

(c)

FIGURE 2: The LST distribution for Shanghai: (a) 1989, (b) 2000, and (c) 2013.

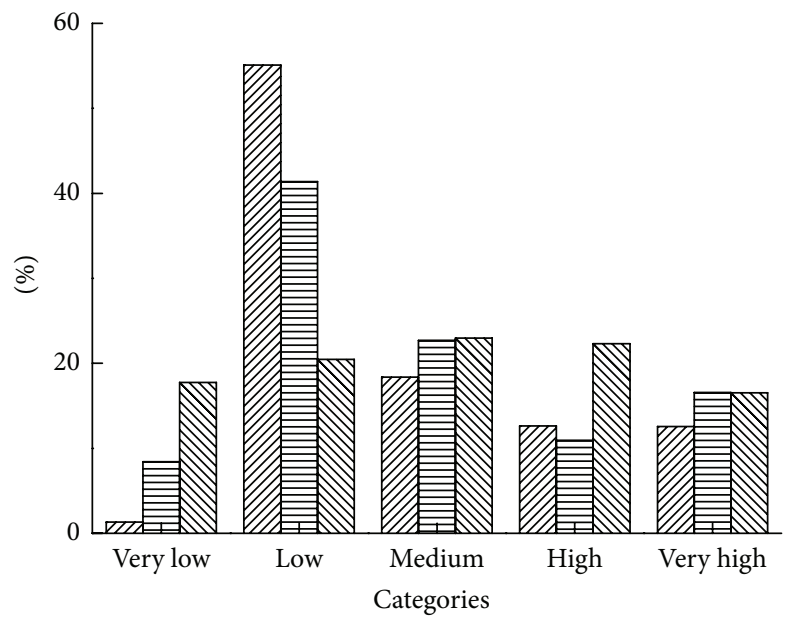

WIIA 1989
E 2000

लाख 2013

FIGURE 3: The histogram of the land area in each LST class.

LST differentials have become more obvious, showing a more diverse distribution towards the two extremes on the LST spectrum: for example, the Dianshan Lake in the west of Qingpu district was primarily categorized as "low" in 1989, because the LST was not significantly lower than city center (within 1 standard deviation from average LST according to Table 1). However for 2000 and 2013, it had shifted to "very low" because the LST difference had increased and become larger than 1 standard deviation from average LST. Similar patterns were also observed in rural areas in Jinshan district and the east of Pudong district along seafront.

The intermediate classes showed a different pattern: areas with high LSTs have increased largely from $12.64 \%$ to $10.93 \%$ to $22.31 \%$, whereas areas with low LSTs have decreased significantly from $55.09 \%$ to $41.38 \%$ to $20.45 \%$. This suggests that the city has been moving to the "hot" side on the LST spectrum.

To further investigate the spatial change of LST over the years, the changes of LST classes between years were calculated (refer to Table 2). Figure 4 shows the patterns of such changes. The patterns are different for different time period. Between 1989 and 2000, the areas with a large LST increase mostly occurred at the urban fringe around the city center, and there was no obvious LST decrease. Between 2000 and 2013, the areas with large increase showed a more scattered pattern which was caused by the urban expansion and industrial development in rural areas including Baoshan, Minhang, Songjiang, Jiading, Fengxian, Jinshan, and Qingpu districts.

In particular, there has been noticeable "large decrease" in the city center. This phenomenon can be attributed to the restriction for development and the establishment of more green spaces in the city center and can be better interpreted within the context of Shanghai's developing strategies and policies for the past decade. At the end of the last century, the Shanghai Municipal Government had issued the "Shanghai City Master Plan 1999-2020," according to which the fundamental principles for city center development were "restricted development" and "sustainable development," that is, to strictly control population density and decrease plot ratio and building volume in city center, and to improve downtown urban greening and open spaces. In particular, the plot ratio for residential buildings was required to be lower than 2.5, and 4.0 for commercial buildings. The "Shanghai City Center Public Green Space Plan" was further issued in 2003 with the objective to increase the green coverage ratio in city center to $35 \%$ by 2020 . The implementation of such policies has a large influence on the urban environment of Shanghai. According to the survey results released by Bureau of Planning, Land and Resources of Shanghai Municipality and published in Shanghai Statistical Yearbook Series for the last decade, by 

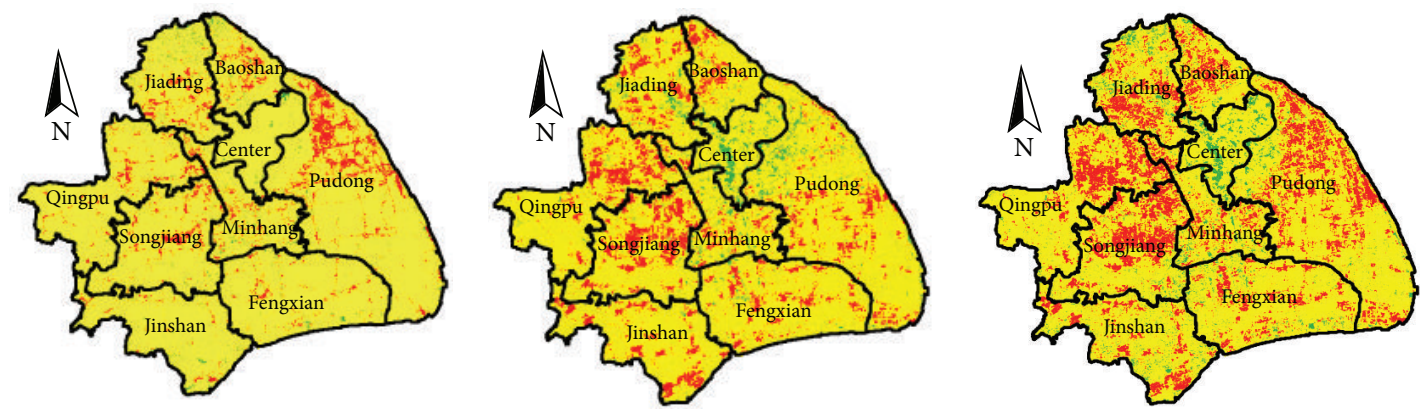

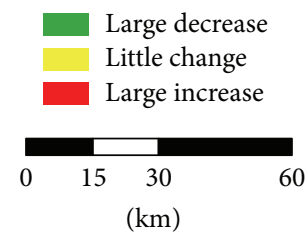

(a)

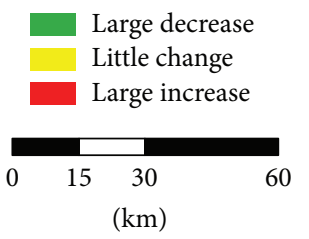

(b)

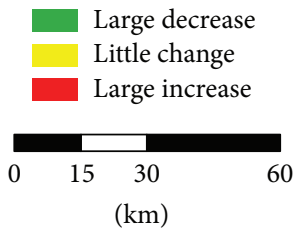

(c)

FIgURE 4: The changes in LST in Shanghai: (a) from 1989 to 2000, (b) from 2000 to 2013, and (c) from 1989 to 2013.

TABLE 3: The LST transition matrix for the changes between different LST classes for each year.

(a)

\begin{tabular}{lccccc}
\hline 1989 & & \multicolumn{2}{c}{2000} \\
& Very low & Low & Medium & High & Very high \\
\hline Very low & 31.75 & 53.80 & 11.39 & 2.21 & 0.85 \\
Low & 13.59 & 59.18 & 18.76 & 5.12 & 3.35 \\
Medium & 2.51 & 36.64 & 39.79 & 13.32 & 7.74 \\
High & 0.33 & 10.02 & 35.36 & 30.74 & 23.55 \\
Very high & 0.17 & 0.55 & 3.39 & 13.90 & 81.99 \\
\hline
\end{tabular}

(b)

\begin{tabular}{lccccc}
\hline \multirow{2}{2}{000} & \multicolumn{5}{c}{2013} \\
& Very low & Low & Medium & High & Very high \\
\hline Very low & 49.01 & 23.19 & 14.35 & 8.47 & 4.98 \\
Low & 28.29 & 29.70 & 21.42 & 12.95 & 7.64 \\
Medium & 6.66 & 21.98 & 34.15 & 24.08 & 13.13 \\
High & 1.90 & 7.51 & 28.25 & 40.01 & 22.33 \\
Very high & 1.13 & 2.37 & 12.38 & 38.63 & 45.49 \\
\hline
\end{tabular}

(c)

\begin{tabular}{lccccc}
\hline 1989 & & \multicolumn{5}{c}{2013} \\
& Very low & Low & Medium & High & Very high \\
\hline Very low & 50.86 & 19.82 & 13.55 & 10.82 & 4.94 \\
Low & 25.89 & 25.30 & 21.72 & 15.96 & 11.12 \\
Medium & 11.66 & 22.87 & 28.35 & 22.57 & 14.55 \\
High & 3.60 & 12.71 & 29.12 & 33.47 & 21.09 \\
Very high & 1.64 & 3.48 & 15.36 & 39.70 & 39.81 \\
\hline
\end{tabular}

the end of 2014 , the green coverage ratio in city center has increased to $38.36 \%$ from $19.1 \%$ in 1998 , and public green
TABLE 4: The area of land that followed the three LST change trends during different periods.

\begin{tabular}{lccc}
\hline Year & \multicolumn{3}{c}{$\begin{array}{c}\text { Area }\left(\mathrm{km}^{2}\right) \\
\text { Little change }\end{array}$} \\
\hline 1989 to 2000 & $118.84(2.28 \%)$ & $4756.18(91.44 \%)$ & $326.62(6.28 \%)$ \\
2000 to 2013 & $268.88(5.17 \%)$ & $4214.32(80.98 \%)$ & $720.22(13.85 \%)$ \\
1989 to 2013 & $352.53(6.78 \%)$ & $3913.43(75.24 \%)$ & $935.57(17.99 \%)$ \\
\hline
\end{tabular}

space per capita has increased to $13.38 \mathrm{~m}^{2}$ from $2.96 \mathrm{~m}^{2}$ in 1998. Meanwhile, only moderate development has been carried out in the city center. Taking the post-Expo years, for example, from 2010 to 2013, the total floor area in the city center has increased from $304,790,000 \mathrm{~m}^{2}$ to $337,470,000 \mathrm{~m}^{2}$, with ca. $10 \%$ increase, which is in contrast with the drastic increase in suburban districts during the same time period, such as $21.2 \%$ increase in Baoshan district, $48.5 \%$ in Jinshan district, and $155.5 \%$ in Fengxian district. In such a case, the restricted development practice and the promotion of green spaces in city center have contributed to the mitigation of LST. A lot of downtown areas have experienced "large decrease" of LST change, resulting in the fact that the city center was no longer the cluster for LST hotspots.

The transition matrix in Table 3 summarizes the pattern of the change between different LST classes between different years. The matrix shows that while most areas stayed for the same LST classes, there was a significant transfer trend to higher LST classes, such as from "very low" to "low," or from "high" to "very high." On the other hand, the transfer to low LST class such as "low" and "very low" was less likely.

Table 4 summarizes the proportions of land areas following the three different LST change trends. During the study period from 1989 to 2013, most of the land (75.24\%) had little change in terms of LST class; however, there is more 

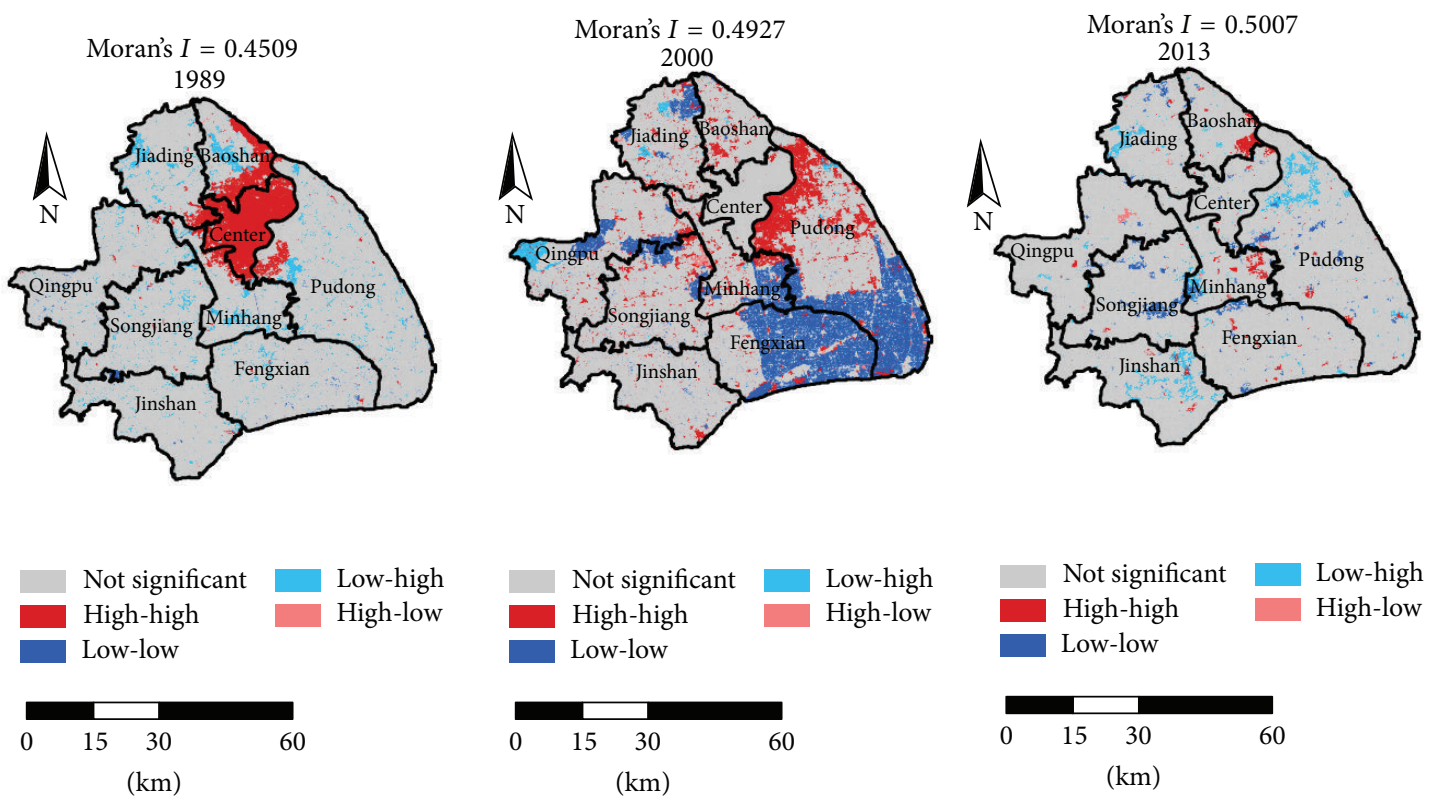

FIGURE 5: Spatial association of the thermal landscape pattern in Shanghai in 1989, 2000, and 2013.

land with large increase of LST (17.99\%) than large decrease $(6.78 \%)$, which confirms that the thermal environment has been "heating up." Also, the area with large increase from 2000 to $2013(13.85 \%)$ was significantly higher than that from 1989 to 2000 (6.28\%), suggesting that this heating up process has been accelerating.

3.2. Spatial Association of LST Classes. Moran's I indicator was calculated to represent spatially the heterogeneity of the thermal pattern of Shanghai, as shown in Figure 5. By definition, the positive value of Moran's $I$ indicates that the LST distribution was random, but with similar LST classes being spatially aggregated. Different years showed different aggregation patterns. For the year of 1989, the predominant high-high aggregation only concentrated in city center, which indicates that areas with high LST were surrounded by similar LST classes. The rest of the districts showed no noticeable aggregation except for scattered low-high aggregation meaning areas with low LST were surrounded by high LST, which mainly occurred in the suburban areas around the local town center, such as Baoshan and Minhang districts. For the year of 2000, both high-high aggregation and lowlow aggregation could be observed. High-high aggregation mainly occurred in Pudong district along the Huangpu River, where intensive development was happening from the early 1990 s to the 2000s. Low-low aggregation occurred in the south of Pudong district and Fengxian district, where the developing speed had been relatively slow. For the year of 2013, no significant aggregations were observed; in contrast, the aggregation pattern was very sparse, which indicates that the whole city has become more homogenous thermally in terms of LST classes.

3.3. Gravity Centroid Movement Analysis of Different LST Classes. To examine the spatial evolution of the thermal environment, the centroids of different LST classes for each year were calculated, as shown in Figure 6. It can be seen that the centroid of very high LST class was right in the city center for 1989 , which is as expected because city center was the predominant areas with very high LST at that time. The centroid has moved southwards for 2000 and 2013. In contrast, the centroid of very low LST class was located in the south part of Shanghai and has moved northwards over the study period. Figure 7 shows the centroid movement paths from 1989 to 2013. Numerical analysis revealed that the biggest centroid movement occurred for the two extreme LST classes for the study period from 1989 to 2013: the centroid for very low LST has moved $3.17 \mathrm{~km}$ to the north, and $5.52 \mathrm{~km}$ to the west; the centroid for very high LST has moved $3.28 \mathrm{~km}$ to the south, and $3.89 \mathrm{~km}$ to the west. Similarly, the centroids for low and high LST classes also showed north-south opposite movements, but with shorter movement distances: the centroid for low LST has moved $1.81 \mathrm{~km}$ to the north, and that for high LST has moved $1.93 \mathrm{~km}$ to the south. They both moved eastwards, $2.46 \mathrm{~km}$ and $2.83 \mathrm{~km}$ for low and high LST classes, respectively. The centroid of medium LST class moved $2.94 \mathrm{~km}$ to the east but did not show obvious movement along the north-south direction, that is, $0.43 \mathrm{~km}$ to the south. The movement pattern could be better explained by relating Figure 2 with Shanghai's urban development over the study period. The most obvious change is the southward movement for very high LST. This occurred because Shanghai has experienced dramatic urban development in the south over the study period, such as Minhang and Fengxian districts. In contrast, the centroid for very low LST moved northward. This is because previously very low LST mainly occurred in the south where there is less intensive development, but over the study period patches of very low LST also emerged in city center and urban fringes in the north. This development trend along the north-south 

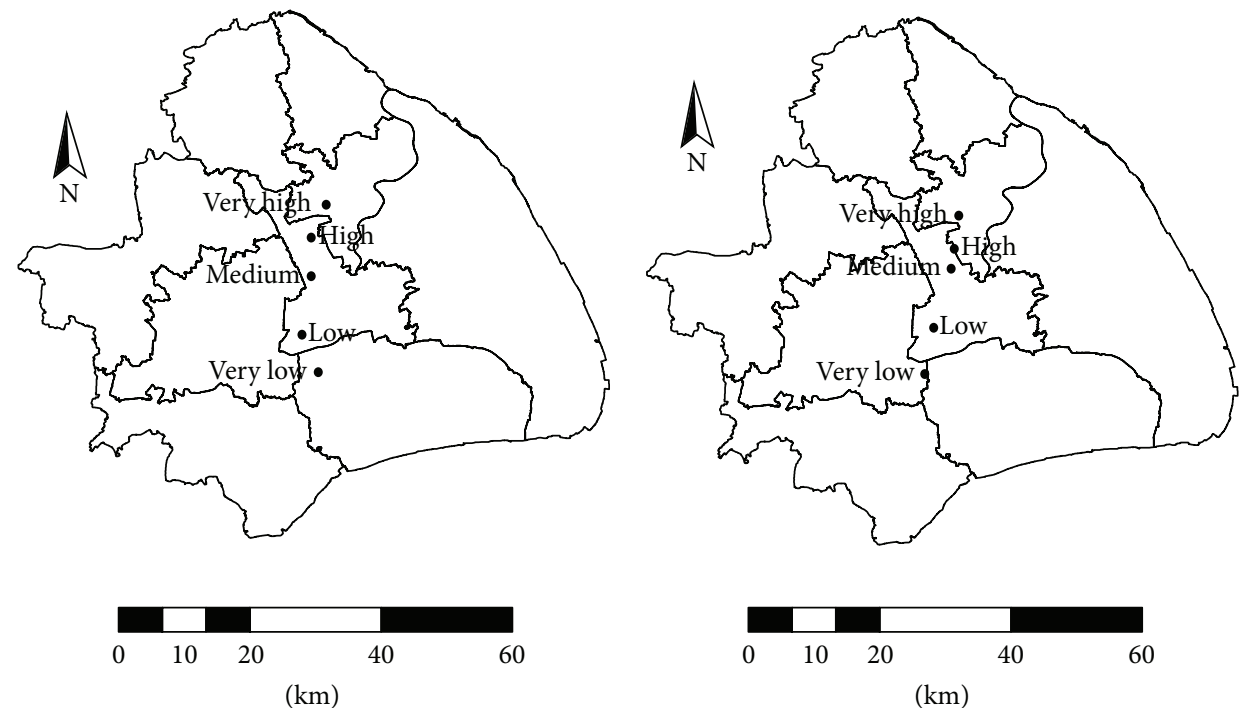

(a)

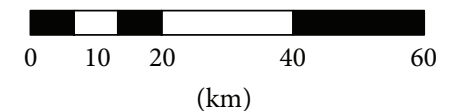

(b)

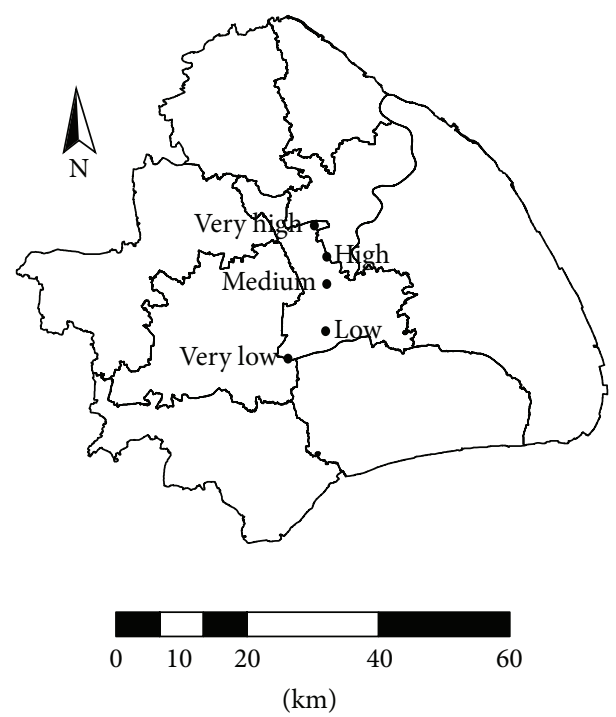

(c)

FIGURE 6: The centroid of different LST classes from 1989 to 2013.

polar also explains the movements of the centroids for high and low LST. Along the east-west dimension, because more patches with very high LST emerged in the west, for example, Songjiang and Qingpu districts, therefore the centroid moved westwards. Meanwhile, the development in Pudong district and the east of Baoshan district resulted in more areas with high LST, which caused its centroid to move eastwards. On the other hand, Qingpu and Songjiang districts in the west had less area with low LST because of the development there, and therefore the centroid for low LST moved eastwards as well. It could be found that overall the LST variation along the north-south dimension was more obvious than that along the east-west dimension. Plus intraurban SUHI variability increased instead of the previous urban-rural difference in 1989, which again confirms the decreasing aggregation pattern as discussed in Section 3.2.

\section{Discussions}

4.1. Trends of SUHI Change in View of Urban Development: Potential Drivers. The spatial analysis shows the pattern and dynamics of the change of Shanghai's SUHI over the study period. The results reveal that the area with high LST distributed in the center of Shanghai and has expanded, primarily to the south. The potential drivers and possible impacts of such SUHI change could be better understood in relation with the socioeconomic characteristics of Shanghai in an urban development context. Previous studies found 


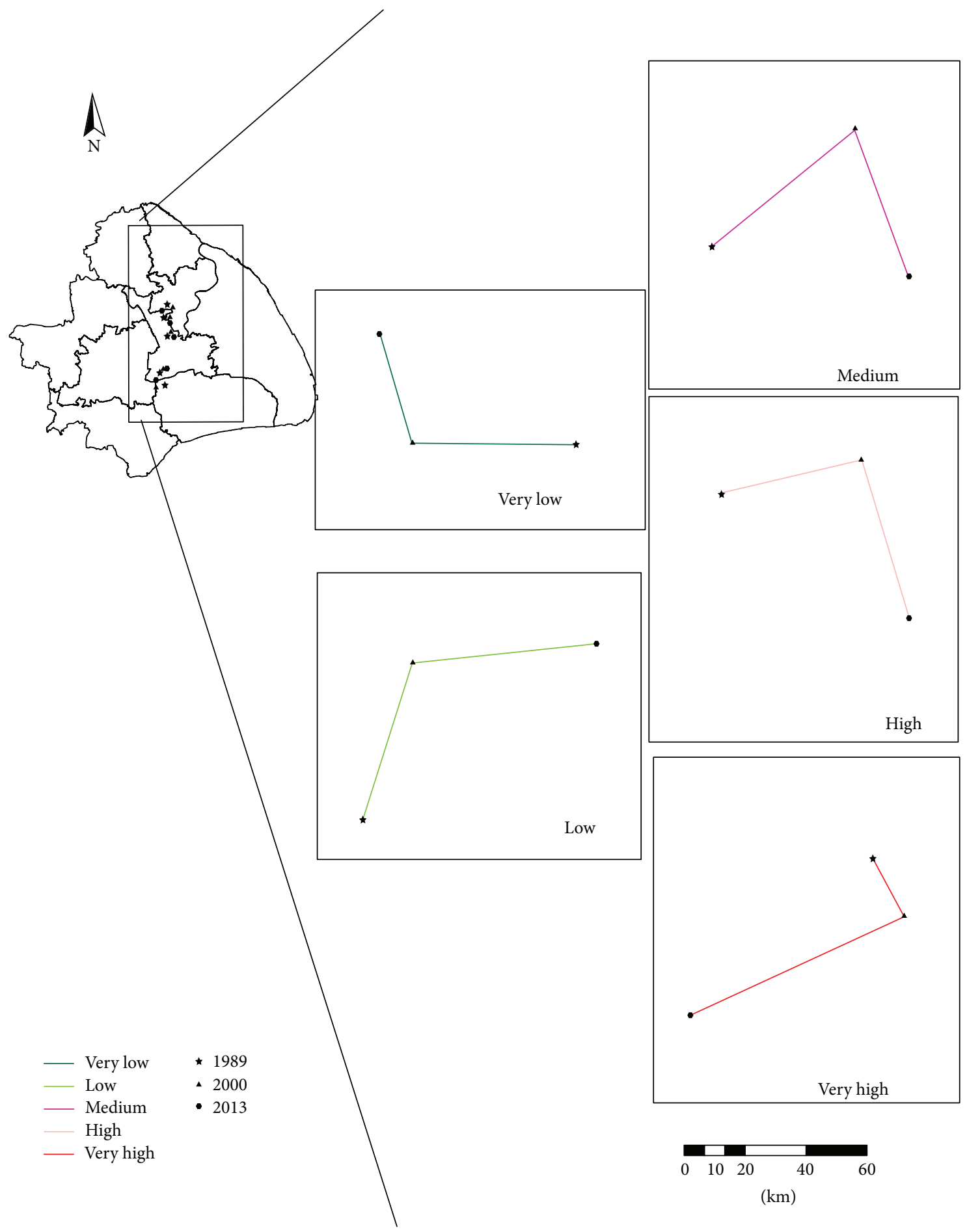

FIGURE 7: The centroid movement of different LST classes from 1989 to 2013.

that the SUHI increased with city sizes and was highly related with land use change $[7,26,34,35]$. To explore the drivers and impacts of the presented SUHI change, this study selected four factors which reflect the level for socioeconomic development of Shanghai, namely, land use, population density, development intensity, and urban greening. Statistical regression methods were used to examine the relationship between LST and the indicators.
4.1.1. Land Use. Three land use types were classified for all the three studied years from Landsat data, namely, water area, urban area, and vegetation. Compared with the single year data used in most studies, data from three years were expected to reflect the temporal characteristic of SUHI change from 1989 to 2013 and the response of the SUHI to urbanization intensity. Land use maps were derived and coupled with the five LST classes, as shown in Figure 8. It 

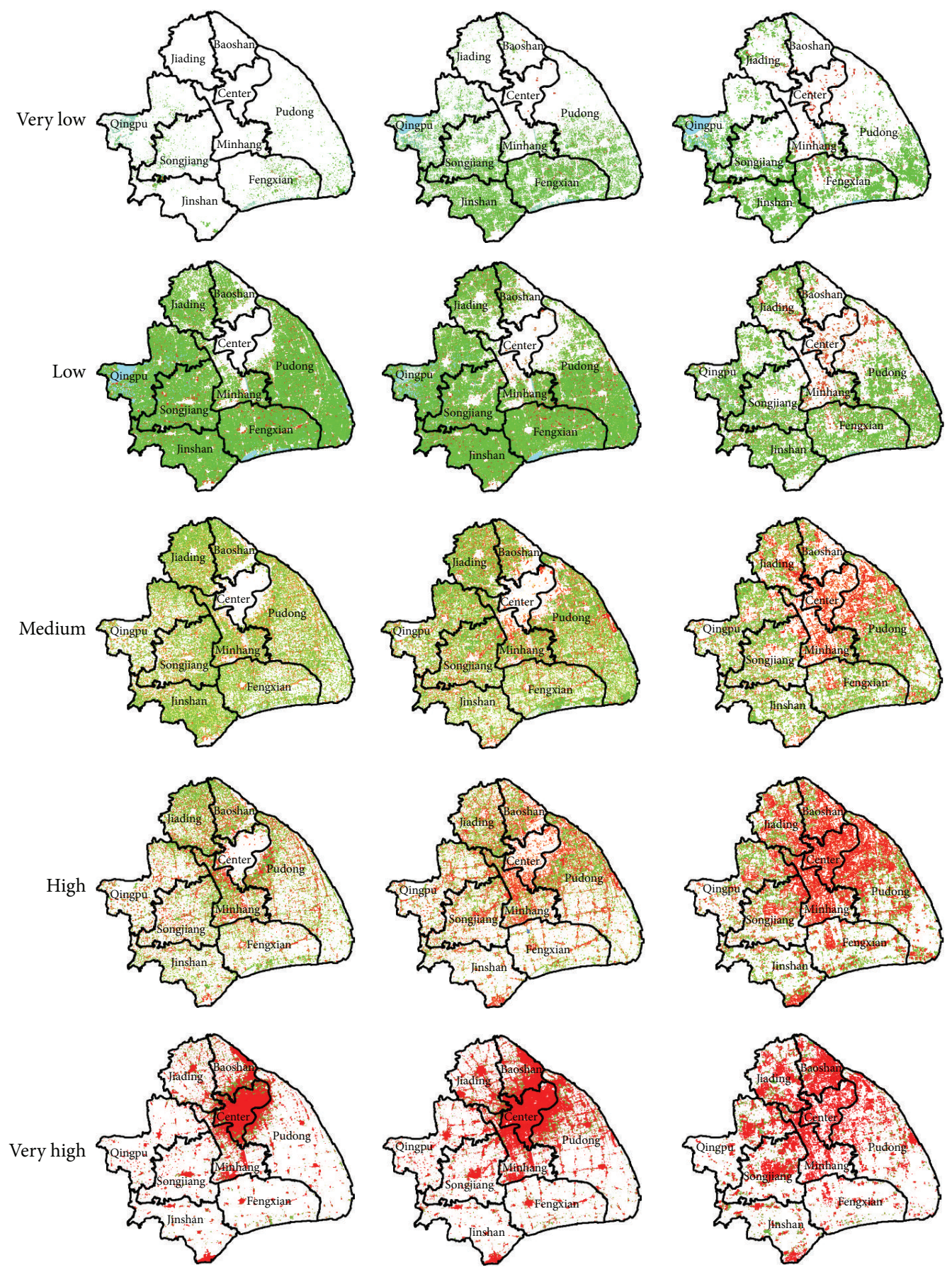

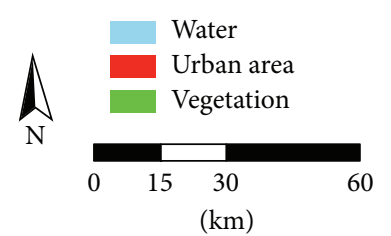

(a)

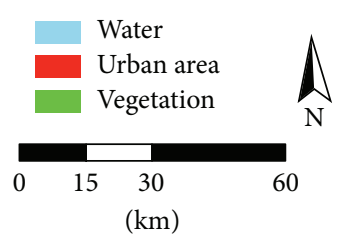

(b)

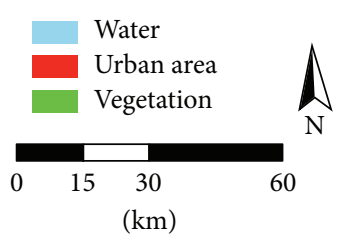

(c)

FIGURE 8: The land use of the 5 LST classes in Shanghai: row 1: very low, row 2: low, row 3: medium, row 4: high, and row 5: very high; (a) 1989, (b) 2000, and (c) 2013. 
is found that areas with very high LST class were primarily urban area, which occupied $84.92 \%, 89.61 \%$, and $86.20 \%$ of the total area in 1989,2000, and 2013, respectively. In contrast, areas with very low LST class were primarily water area and vegetation, which together occupied 97.69\%, 98.39\%, and $95.42 \%$ in 1989,2000 , and 2013 , respectively. Similar findings were also found for high and low LST classes. The high proportion of urban area in high LST classes and high proportion of vegetation in low LST classes indicate that that the expansion of urban area and the loss of vegetation are the most important driver for SUHI change, which is in accordance with findings in [26]. Moreover, by examining between different years and relating Figure 2, it is affirmative that the expansion of urban area has enhanced the SUHI, as indicated by the increasing proportion of urban area in high and very high LST classes.

4.1.2. Population Density. Population density data for Shanghai's 16 administrative districts were collected from Shanghai Statistical Yearbook (1990, 2001, and 2014). Spatial average LSTs for all the districts were calculated. Regression analysis revealed that there was a significant positive exponential correlation between LST and population density with high $R$-square and Pearson correlation coefficient, as shown in Figure 9. The decreasing order of Pearson correlation coefficient was $0.796(2000)>0.743(2013)>0.661$ (1989) (all significant at 0.01 level). The positive correlation between LST and population density was in accordance with findings in $[36,37]$ and confirms that city size as indicated by population density is one of the important drivers for SUHI increase. The regression coefficient of 0.592 for year 2013 is comparable to the regression coefficients of 0.633 found in a previous study which used 2008 data [36]. On the other hand, the regressions also show temporal differences. The regression coefficient has decreased largely from 1.586 in 2000 to 0.592 in 2013. This suggests that population density has a decreasing impact on SUHI as the city becomes more urbanized. Before the 1990s there was an obvious urban-rural difference, where more populated city center had LST significantly higher than rural areas with lower population density, as shown in Figure 2. At that time population density was a primary driver and an important indicator for SUHI intensity. However, after 2000 , because of the restrictive development strategies and mitigation measures implemented in city center (the readers are also referred to Section 3.1 for discussions on development policies), and the extensive new town development in less populated urban fringes and suburban areas, the SUHI intensity between city center and rural and suburban areas was not as significant as the difference in population density. Similar findings were also reported in [26], where the regression coefficient (linear) for population density in correlation with SUHI intensity has decreased from 0.005 in 2000 to 0.002 in 2008.

4.1.3. Development Intensity. One limitation of using population data from statistical yearbooks to reflect the city size is that they only consider residential population density. To examine the "actual" development intensity of a city, commercial and industrial activities also need to be taken into account. In this sense, nighttime stable light data from the Defense Meteorological Satellite Program's Operational Line-Scan System (DMSP-OLS) was used to characterize the city size and development intensity (e.g., [18]). For the comparison over time series data from multiple satellites, the original data was corrected and the details for data correction followed the procedures used in [38]. Regression analysis showed that there was a significant linear positive correlation between LST and DMSP, as shown in Figure 10. The Pearson correlation coefficients between temperature and DMSP in 2000 and 2013 were 0.961 and 0.950 , respectively (both at 0.01 significant level). The results showed that urban development intensity, primarily human activities, is strongly related to the increase of SUHI intensity. Similar to findings in the discussion on population density, the regression coefficient has also decreased from 0.135 in 2000 to 0.075 in 2013, confirming that the role development intensity plays in affecting SUHI intensity has decreased as the city becomes more urbanized. The findings are in agreement with previous studies; for example, in [26], the linear regression coefficient for ratio of developed land in correlation with SUHI intensity decreased from 0.34 in 2001 to 0.29 in 2005.

4.1.4. Urban Greening. The urban greening of Shanghai was characterized by the Normalized Difference Vegetation Index (NDVI) which was derived from Landsat ETM+/OLS images based on conventional routines (e.g., [27, 39]). Spatial average of LST and NDVI for all 16 administrative districts was calculated. Regression analysis revealed that there was a strong negative linear correlation between mean LST and NDVI, as shown in Figure 11. The Pearson correlation coefficient was $-0.977,-0.978$, and -0.876 in 1989, 2000, and 2013, respectively (all significant at 0.01 level). The regression showed that urban greening has a mitigating effect to the SUHI in Shanghai, which supports the mechanism that vegetation can increase the latent heat fluxes via transpiration and effectively cool surface temperature $[15,16,28,40]$. Compared with the decreasing impact of population density and development intensity as drivers for SUHI intensity, NDVI did not show much decrease, with the negative regression coefficient of -18.380 in $1989,-13.467$ in 2000 , and -12.296 in 2013. This suggests that as the city becomes more urbanized, urban greening constantly has a significant mitigating effect to SUHI for different development stages.

4.2. Bridging the Local and the Holistic Scales. In the present study, two spatial analysis methods were applied to analyze the spatial structure of the SUHI of Shanghai, namely, spatial association and centroid movement analysis. The two methods worked at local and holistic scales, respectively, and collaboratively characterized the pattern and dynamics of the spatiotemporal evaluation of the SUHI of Shanghai. The spatial association of different LST classes interpreted the spatial aggregation pattern of different SUHI intensity and showed the intraurban variability of SUHI in Shanghai from the local scale. For example, the emergence of high-high LST aggregation in Pudong district in 2000 can be largely attributed to the intensive urban development at that time. In comparison, the centroid movement method analyzed the 

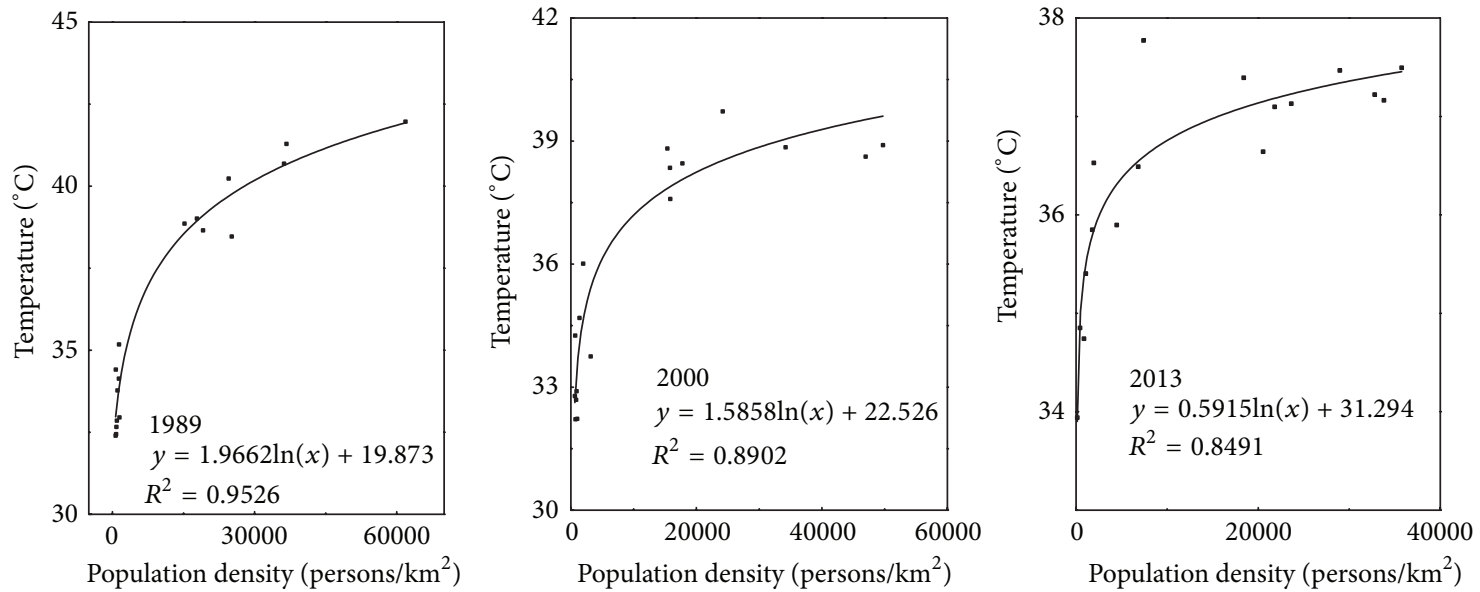

FIGURE 9: Correlation between LST and population density ( $x$ : population density; $y$ : mean LST).
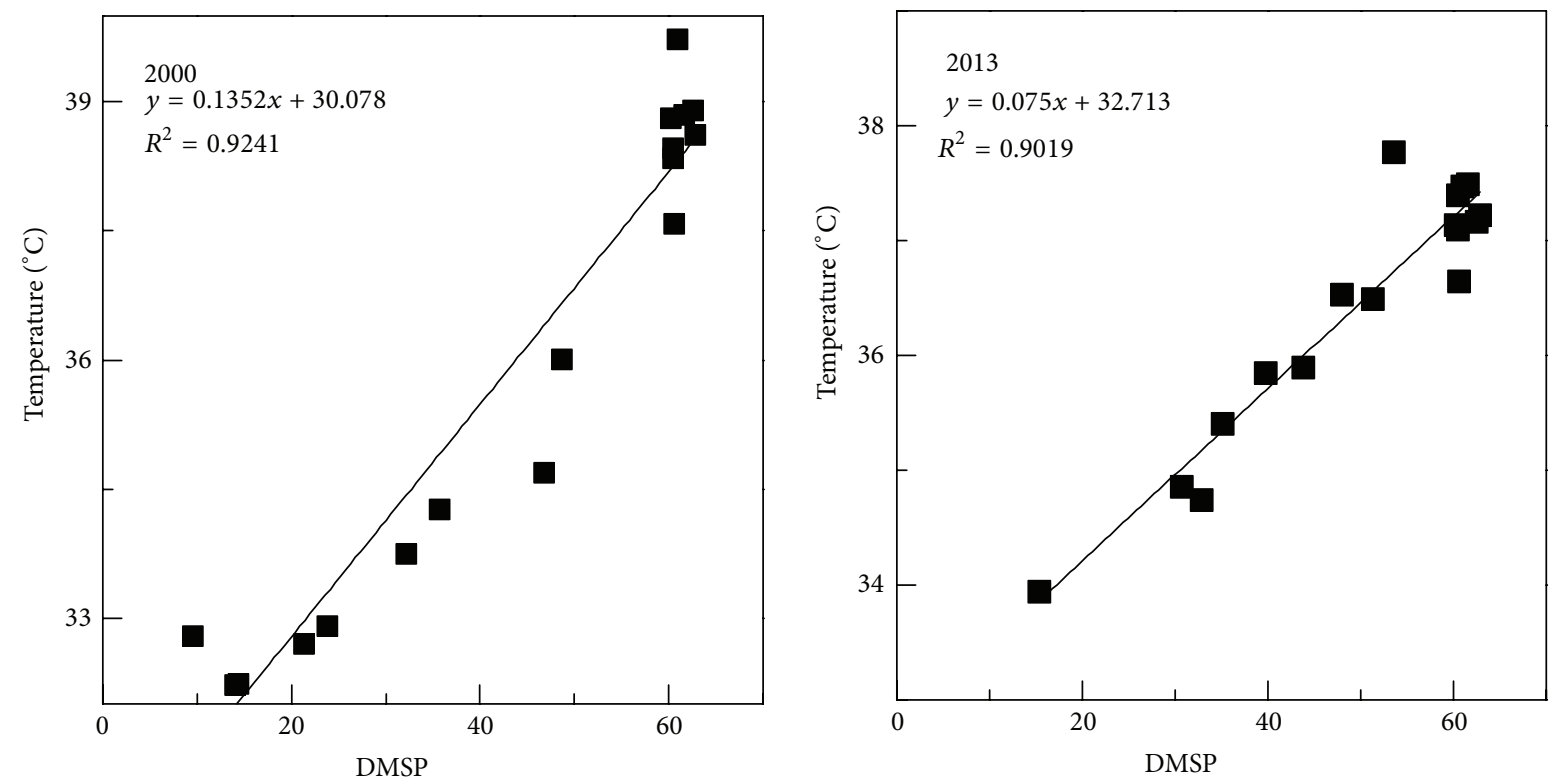

Figure 10: Correlation between LST and DMSP ( $x$ : mean DMSP; $y$ : mean LST).
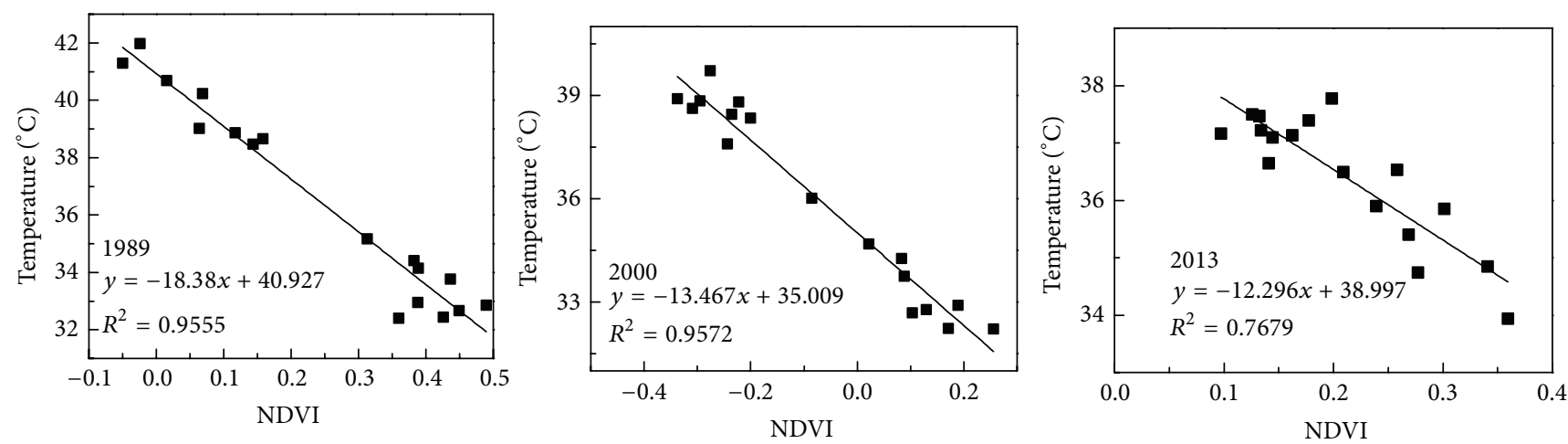

FIGURE 11: Correlation between LST and NDVI ( $x$ : mean NDVI; $y$ : mean LST). 
movement paths and distances of the centroids of different LST classes and showed the spatial change trends of SUHI from the holistic scale. The two methods together revealed that Shanghai's SUHI has evolved dramatically for the last 20 years. There is a significant increase of areas with high LST; on the other hand the local aggregation of high LST has largely decreased, indicating that the whole city is heating up and becomes more homogenous instead of the previous urban-rural SUHI mechanism. Also, such heating up process has developed more along the north-south dimension, that is, with the emerging "hotspots" moving southwards, which is in accordance with the urban development process of the city. The understanding of the spatiotemporal evolution of SUHI from both local and holistic scales is essential to examine drivers and impacts of the present SUHI pattern and dynamics and is only possible with the aid of remote sensing techniques. This study demonstrates that these spatial analysis methods can be jointly adopted to characterize SUHI evolution both qualitatively and quantitatively.

4.3. Implications for Urban Planning. The study shows that SUHI is an important indicator to describe the urban thermal environment. The spatiotemporal characteristics of SUHI change are helpful for understanding urban ecology as well as land use planning to minimize the potential negative impacts imposed by urbanization. For example, rapid urbanization has resulted in massive impervious urban surface, which contributes largely to the deterioration of the urban thermal environment. The study showed that the development of suburban areas in Shanghai, such as Minhang, Jiading, Baoshan, Songjiang, and Qingpu districts, had caused large increase of SUHI intensity in these areas. It is also found that such adverse impacts could be mitigated by altering the spatial configuration of different land use and functional zones. For example, spatial analysis revealed that hotspots of the city have sprawled from the city center to urban fringe and suburban areas, and a lot of local hotspots emerged in new towns in Jiading, Songjiang, and Qingpu districts where large areas of vegetation have been converted to imperious surface. This suggests that development in these districts should be carried out with moderation. On the other hand, low SUHI patches occurred in city center, especially in the year of 2013. This could be attributed to the increase of urban greening and open spaces in city center for the last decade, a period when Shanghai's urban redevelopment was largely influenced by the Expo event. The strategy of embedding such green patches in city center has been proved to be an effective way to mitigate SUHI $[28,41]$. Meanwhile, these areas are already very fragile thermally, as indicated by the predominant high LST; therefore no further development should be carried out; instead actions to improve the local thermal environment need to be taken, such as improving energy efficiency, using environmentally friendly building materials, and taking other ecological measures.

\section{Conclusions}

This paper investigated the spatiotemporal evolution of the SUHI in Shanghai from 1989 to 2013, a period during which the city has experienced drastic urban development. Remote sensing techniques were applied to retrieve LST from Landsat images. The SUHI intensity was quantified into 5 classes of LST value, ranging from very low to very high. Spatial analyses including spatial association and centroid movement analysis were carried out to look at the pattern and dynamics of SUHI change. It is found that, during the study period, Shanghai's SUHI has become more severe; that is, the whole city has been heating up, as indicated by the large increase of areas with high and very high LST. Spatially, the SUHI intensity has developed more significantly along the north-south direction, which is in accordance with the city's intensive development of new towns in districts including Baoshan, Minhang, Songjiang, and Fengxian. In terms of the relationship between SUHI and urban development, possible drivers of such SUHI change trends were discussed. Factors including land use change, population density, development intensity, and urban greening were examined by statistical analysis methods. It is shown that land use change, that is, converting vegetation to built-up area, is the most important factor that contributes to the increase of SUHI intensity. Moreover, population density and development intensity as described by nighttime light data both showed strong positive correlation with the increase of SUHI intensity, whereas urban greening was shown to have effective cooling functions of the thermal environment. This study also showed that the two spatial analysis methods employed could collaboratively link the local and holistic scales for the analysis of urban thermal environment and contribute to comprehensive understanding of the drivers and impacts of SUHI change which can lead to more effective planning strategies towards sustainable urban development.

\section{Highlights}

(1) The patterns and dynamics of the SUHI change in Shanghai from 1989 to 2013 were investigated from both local and holistic scales and related with urban development especially in the pre- and post-Shanghai Expo era.

(2) The study reveals that the SUHI intensity in Shanghai has developed more significantly along the northsouth dimension, which is in agreement with local urban development activities.

(3) SUHI intensity in city center has shown to be mitigated for the past decade, because of the implementation of more urban green spaces as directed by planning strategies and development policies.

\section{Conflict of Interests}

The authors declare that there is no conflict of interests regarding the publication of this paper.

\section{Acknowledgments}

This study is supported by the National Natural Science Foundations of China no. 41301087, no. 41271055 and China Postdoc Foundation no. 2013M540346. The authors would 
like to thank the reviewer for the valuable and pertinent review comments which have helped to largely improve the paper.

\section{References}

[1] UNFPA, State of World Population 2009_Facing a Changing World: Women, Population and Climate, United Nations Population Fund (UNPFA), New York, NY, USA, 2009.

[2] L. O. Myrup, "A numerical model of the urban heat island," Journal of Applied Meteorology, vol. 8, no. 6, pp. 908-918, 1969.

[3] A. J. Arnfield, "Two decades of urban climate research: a review of turbulence, exchanges of energy and water, and the urban heat island," International Journal of Climatology, vol. 23, no. 1, pp. 1-26, 2003.

[4] T. R. Oke, "City size and the urban heat island," Atmospheric Environment, vol. 7, no. 8, pp. 769-779, 1973.

[5] P. Gong, S. Liang, E. J. Carlton et al., "Urbanisation and health in China," The Lancet, vol. 379, no. 9818, pp. 843-852, 2012.

[6] J. O'Loughlin, F. D. W. Witmer, A. M. Linke, A. Laing, A. Gettelman, and J. Dudhia, "Climate variability and conflict risk in East Africa, 1990-2009," Proceedings of the National Academy of Sciences of the United States of America, vol. 109, no. 45, pp. 18344-18349, 2012.

[7] M. L. Imhoff, P. Zhang, R. E. Wolfe, and L. Bounoua, "Remote sensing of the urban heat island effect across biomes in the continental USA," Remote Sensing of Environment, vol. 114, no. 3, pp. 504-513, 2010.

[8] D. R. Streutker, "Satellite-measured growth of the urban heat island of Houston, Texas," Remote Sensing of Environment, vol. 85, no. 3, pp. 282-289, 2003.

[9] J. A. Voogt and T. R. Oke, "Thermal remote sensing of urban climates," Remote Sensing of Environment, vol. 86, no. 3, pp. 370384, 2003.

[10] P. K. Rao, "Remote sensing of urban 'heat islands' from an environmental satellite," Bulletin of the American Meteorological Society, vol. 53, pp. 647-648, 1972.

[11] T. N. Carlson, J. A. Augustine, and F. E. Boland, "Potential application of satellite temperature measurements in the analysis of land use over urban areas," Bulletin of the American Meteorological Society, vol. 58, pp. 1301-1303, 1977.

[12] J. C. Price, "Assessment of the urban heat island effect through the use of satellite data," Monthly Weather Review, vol. 107, no. 11, pp. 1554-1557, 1979.

[13] W. H. Carnahan and R. C. Larson, "An analysis of an urban heat sink," Remote Sensing of Environment, vol. 33, no. 1, pp. 65-71, 1990.

[14] K. P. Gallo, A. L. McNab, T. R. Karl, J. F. Brown, J. J. Hood, and J. D. Tarpley, "The use of NOAA AVHRR data for assessment of the urban heat island effect," Journal of Applied Meteorology, vol. 32, no. 5, pp. 899-908, 1993.

[15] H. Tran, D. Uchihama, S. Ochi, and Y. Yasuoka, "Assessment with satellite data of the urban heat island effects in Asian mega cities," International Journal of Applied Earth Observation and Geoinformation, vol. 8, no. 1, pp. 34-48, 2006.

[16] S. S. Peng, S. L. Piao, P. Ciais et al., "Surface urban heat island across 419 global big cities," Environmental Science and Technology, vol. 46, no. 2, pp. 696-703, 2012.

[17] A. Benali, A. C. Carvalho, J. P. Nunes, N. Carvalhais, and A. Santos, "Estimating air surface temperature in Portugal using
MODIS LST data," Remote Sensing of Environment, vol. 124, pp. 108-121, 2012.

[18] D. Zhou, S. Zhao, S. Liu, L. Zhang, and C. Zhu, "Surface urban heat island in China's 32 major cities: spatial patterns and drivers," Remote Sensing of Environment, vol. 152, pp. 51-61, 2014.

[19] J. E. Nichol, W. Y. Fung, K.-S. Lam, and M. S. Wong, "Urban heat island diagnosis using ASTER satellite images and 'in situ' air temperature," Atmospheric Research, vol. 94, no. 2, pp. 276284, 2009.

[20] Z. Chen, C. Gong, J. Wu, and S. Yu, "The influence of socioeconomic and topographic factors on nocturnal urban heat islands: a case study in Shenzhen, China," International Journal of Remote Sensing, vol. 33, no. 12, pp. 3834-3849, 2012.

[21] J. C. Jiménez-Munoz and J. A. Sobrino, "A generalized singlechannel method for retrieving land surface temperature from remote sensing data," Journal of Geophysical Research D: Atmospheres, vol. 108, no. 22, pp. 4688-4697, 2003.

[22] M. Stathopoulou, C. Cartalis, and I. Keramitsoglou, "Mapping micro-urban heat islands using NOAA/AVHRR images and CORINE land cover: an application to coastal cities of Greece," International Journal of Remote Sensing, vol. 25, no. 12, pp. 23012316, 2004.

[23] J. A. Sobrino, J. C. Jiménez-Muñoz, J. El-Kharraz, M. Gómez, M. Romaguera, and G. Sòria, "Single-channel and two-channel methods for land surface temperature retrieval from DAIS data and its application to the Barrax site," International Journal of Remote Sensing, vol. 25, no. 1, pp. 215-230, 2004.

[24] P. Dash, F.-M. Göttsche, F.-S. Olesen, and H. Fischer, "Land surface temperature and emissivity estimation from passive sensor data: theory and practice-current trends," International Journal of Remote Sensing, vol. 23, no. 13, pp. 2563-2594, 2002.

[25] Z. Wan, Y. Zhang, Q. Zhang, and Z.-L. Li, "Validation of the land-surface temperature products retrieved from Terra Moderate Resolution Imaging Spectroradiometer data," Remote Sensing of Environment, vol. 83, no. 1-2, pp. 163-180, 2002.

[26] H. Zhang, Z.-F. Qi, X.-Y. Ye, Y.-B. Cai, W.-C. Ma, and M.$\mathrm{N}$. Chen, "Analysis of land use/land cover change, population shift, and their effects on spatiotemporal patterns of urban heat islands in metropolitan Shanghai, China," Applied Geography, vol. 44, pp. 121-133, 2013.

[27] J.-J. Li, X.-R. Wang, X.-J. Wang, W.-C. Ma, and H. Zhang, "Remote sensing evaluation of urban heat island and its spatial pattern of the Shanghai metropolitan area, China," Ecological Complexity, vol. 6, no. 4, pp. 413-420, 2009.

[28] J. Li, C. Song, L. Cao, F. Zhu, X. Meng, and J. Wu, "Impacts of landscape structure on surface urban heat islands: a case study of Shanghai, China," Remote Sensing of Environment, vol. 115, no. 12, pp. 3249-3263, 2011.

[29] B. L. Markham and J. L. Barker, "Spectral characterization of the LANDSAT thematic mapper sensors," International Journal of Remote Sensing, vol. 6, no. 5, pp. 697-716, 1985.

[30] J. Jiménez-Muñoz, J. Cristóbal, J. Sobrino et al., "Revision of the sigle-channel algorithm for land surface temperature retrieval from Landsat theral-infrared data," IEEE Transactions on Geoscience and Remote Sensing, vol. 47, no. 1, pp. 339-349, 2009.

[31] L. Anselin, "Local indicators of spatial association-LISA," Geographical Analysis, vol. 27, no. 2, pp. 93-115, 1995.

[32] H. Y. Ding and W. Z. Shi, "Land-use/land-cover change and its influence on surface temperature: a case study in Beijing City," International Journal of Remote Sensing, vol. 34, no. 15, pp. 55035517, 2013. 
[33] F. H. Wang, Quantitative Methods and Application in GIS, CRC Press, LLC, 2006.

[34] J. E. Nichol, "High-resolution surface temperature patterns related to urban morphology in a tropical city: a satellite-based study," Journal of Applied Meteorology, vol. 35, no. 1, pp. 135-146, 1996.

[35] D. R. Streutker, "A remote sensing study of the urban heat island of Houston, Texas,' International Journal of Remote Sensing, vol. 23, no. 13, pp. 2595-2608, 2002.

[36] Y.-Y. Li, H. Zhang, and W. Kainz, "Monitoring patterns of urban heat islands of the fast-growing Shanghai metropolis, China: using time-series of Landsat TM/ETM+ data," International Journal of Applied Earth Observation and Geoinformation, vol. 19, no. 1, pp. 127-138, 2012.

[37] Y. Hirano and T. Fujita, "Evaluation of the impact of the urban heat island on residential and commercial energy consumption in Tokyo," Energy, vol. 37, no. 1, pp. 371-383, 2012.

[38] Z. Liu, C. He, Q. Zhang, Q. Huang, and Y. Yang, "Extracting the dynamics of urban expansion in China using DMSP-OLS nighttime light data from 1992 to 2008," Landscape and Urban Planning, vol. 106, no. 1, pp. 62-72, 2012.

[39] A. Buyantuyev, J. Wu, and C. Gries, "Multiscale analysis of the urbanization pattern of the Phoenix metropolitan landscape of USA: time, space and thematic resolution," Landscape and Urban Planning, vol. 94, no. 3-4, pp. 206-217, 2010.

[40] F. Yuan and M. E. Bauer, "Comparison of impervious surface area and normalized difference vegetation index as indicators of surface urban heat island effects in Landsat imagery," Remote Sensing of Environment, vol. 106, no. 3, pp. 375-386, 2007.

[41] Q. Weng and S. Yang, "Managing the adverse thermal effects of urban development in a densely populated Chinese city," Journal of Environmental Management, vol. 70, no. 2, pp. 145156,2004 

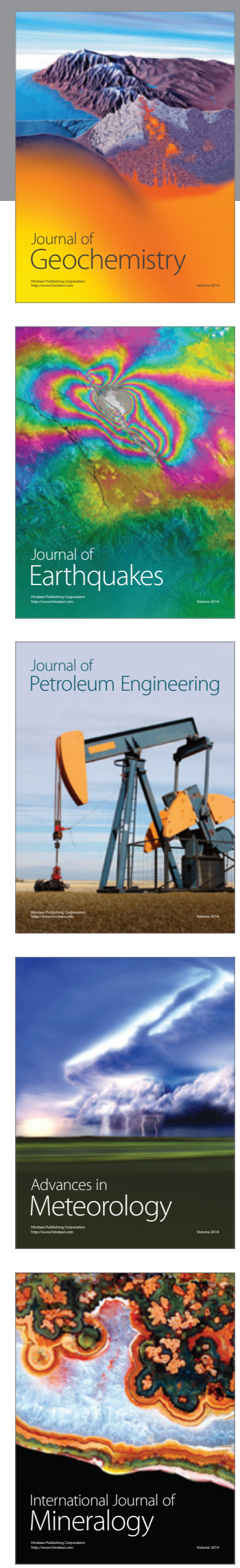
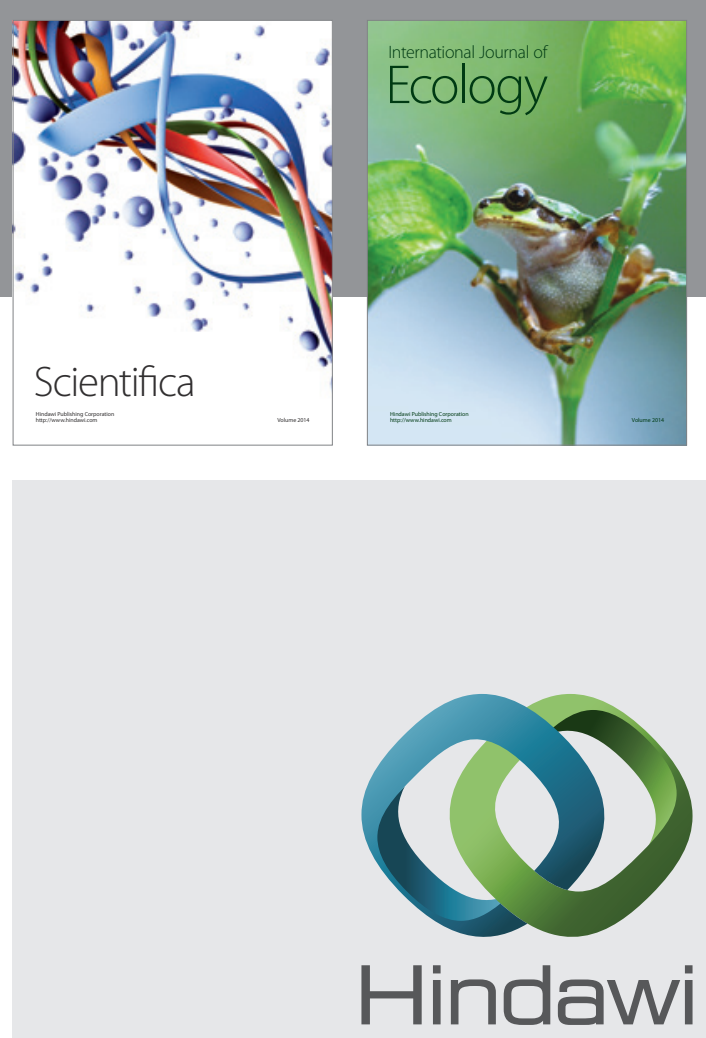

Submit your manuscripts at

http://www.hindawi.com
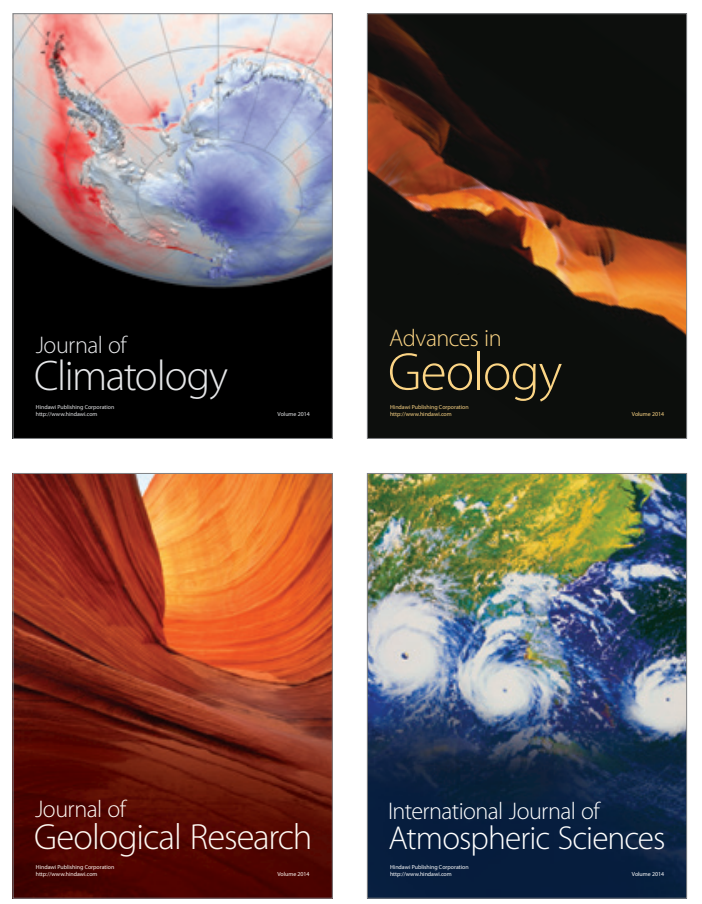

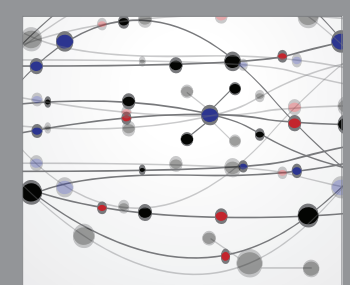

The Scientific

\section{World Journal}
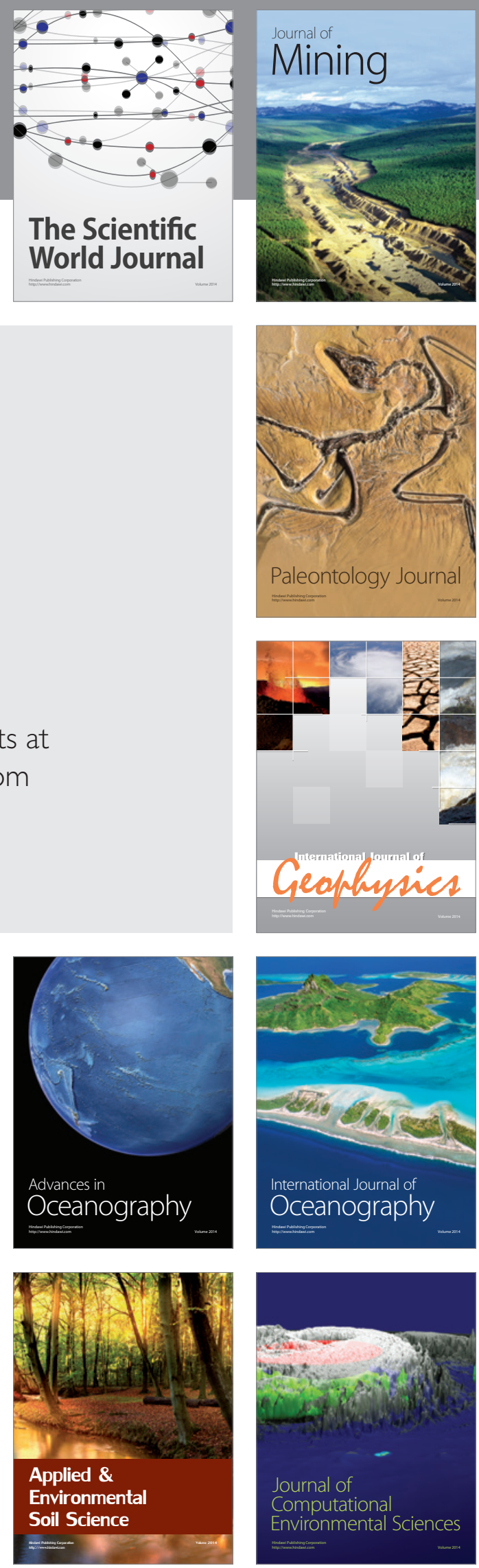